\title{
On the Bertini theorem in arbitrary characteristic
}

\author{
Zbigniew Jelonek
}

Received: 9 May 2012 / Accepted: 5 September 2012 / Published online: 29 September 2012

(C) The Author(s) 2012. This article is published with open access at Springerlink.com

\begin{abstract}
We give a simple direct proof of the Kleiman Bertini theorem in arbitrary characteristic. We also give a simple proof of Serre splitting theorem.
\end{abstract}

Keywords Bertini theorem $\cdot$ Serre splitting theorem

Mathematics Subject Classification (1991) 14 A

\section{Introduction}

Kleiman in the paper [4] proved Bertini theorem in arbitrary characteristic (Corollary 12 in [4]). Kleiman deduced this theorem from his general transversality theorem: if $X$ is $G$-homogenous algebraic variety and $Z, W$ are smooth varieties over $X$, then the fiber product of $Z$ and a general translate $g Y$ over $X$ is smooth. This theorem is valid in characteristic zero, however Kleiman shows that his result still works in positive characteristic, if we additionally assume that the action of the group $G$ is sufficiently good (this assumption is satisfied for the action of the group $P G L(n)$ on the projective space $\mathbb{P}^{n}$ ) and that $Z, W$ are unramified over $X$.

The aim of this note is to give a simple, direct proof of the Bertini Theorem in arbitrary characteristic. We also partially generalize our method to locally free sheafs of higher ranks (mainly for char $k=0$ ). In particular we give a simple proof of the

Communicated by A. Constantin.

The author acknowledge a support of Polish Ministry of Science and Higher Education grant MNiSW N N201 413139, 2010-2013.

Z. Jelonek (凶)

Instytut Matematyczny PAN, ul. Śniadeckich 8, 00-956 Warsaw, Poland

e-mail: najelone@cyf-kr.edu.pl 
fact that if a variety $X$ is smooth and $\mathcal{F}$ is a locally free sheaf on $X$, with sufficiently many sections, then a generic section of $\mathcal{F}$ is transversal to $X$. As a Corollary we give a proof of the Atiyah-Serre Splitting Theorem (compare with [6], [1] and [5]).

\section{Notations and definitions}

We assume for simplicity, that the base field $k$ is algebraically closed. Let $X$ be algebraic variety and $\mathcal{F}$ be a locally free sheaf on $X$ of rank $r$. Take a section $s \in \Gamma(X, \mathcal{F})$. We describe a scheme of zeroes of $s^{-1}(0)$ in the following way: locally we can assume that $X=U$ is an affine variety and $\mathcal{F}=\mathcal{O}_{U}^{r}$ is trivial. Hence $s=\left(s_{1}, \ldots, s_{r}\right)$ where $s_{i} \in \Gamma\left(U, O_{U}\right)=k[U]$. Then $s^{-1}(0)$ is given by the ideal $\left(s_{1}, \ldots, s_{r}\right) \subset k[U]$. We say that the section $s$ is transversal to $X$ if either $s^{-1}(0)$ is smooth and it has dimension $\operatorname{dim} X-r$ or $s^{-1}(0)=\emptyset$.

Let $X, Y$ be smooth varieties and $f: X \rightarrow Y$ be a morphism. We say that $f$ is unramified, if $f$ separates infinitely near points of $X$, i.e., the mapping $d_{x} f: T_{x} X \rightarrow$ $T_{f(x)} Y$ is a monomorphism for every closed point $x \in X$ (see [3], p. 15).

If $X$ is an affine variety we will denote by $k[X]$ the $\operatorname{ring} \Gamma\left(X, O_{X}\right)$. If $M$ is a $k[X]$ module then by $M^{\sim}$ we denote the sheafification of $M-$ see [2], Definition on the page 110 .

\section{Main result}

Theorem 3.1 (Bertini theorem in arbitrary characteristic) Let X be a smooth algebraic variety of dimension $d$ and let $\mathcal{F}$ be an invertible sheaf on $X$. Assume that $\mathcal{F}$ is generated by global sections $s_{1}, \ldots, s_{r} \in \Gamma(X, \mathcal{F})$. Assume that the morphism $\Phi$ from $X$ to the projective space $\mathbb{P}^{r-1}$ given by $s_{1}, \ldots, s_{r}$ is unramified. Then there is a Zariski open non-empty subset $U \subset k^{r}$ such that for every $c=\left(c_{1}, \ldots, c_{r}\right) \in U$ the zero set of the section $s=\sum_{i=1}^{r} c_{i} s_{i}$ is smooth.

Proof Since $X$ is quasi-compact we can assume that $X$ is affine and the sheaf $\mathcal{F}$ is trivial. Hence we can identify $\mathcal{F}$ with $\mathcal{O}_{X}$ and now $s_{i}: X \rightarrow k$ are regular functions. Additionally we can assume that $s_{r} \equiv 1$. Indeed, since $s_{1}, \ldots, s_{r}$ generates $\mathcal{F}$ we have that open (affine!) subsets $U_{i}:=X \backslash\left\{s_{i}=0\right\}$ cover $X$. Consequently we can assume that $s_{r} \neq 0$ in $X$. Take $s_{i}^{\prime}=s_{i} / s_{r}, i=1, \ldots, r$. If we prove our theorem for $s_{i}^{\prime}$, then we automatically prove it also for originals $s_{i}$. Indeed, note that for a fixed $c=\left(c_{1}, \ldots, c_{r}\right) \in k^{r}$ the ideals $\left(\sum_{i=1}^{r} c_{i} s_{i}\right)$ and $\left(\sum_{i=1}^{r} c_{i} s_{i}^{\prime}\right)$ are equal in $k[X]$. Moreover, we can assume that on $X$ we have global local coordinates $x_{1}, \ldots, x_{d}$, i.e., the mapping $\left(x_{1}, \ldots, x_{d}\right): X \rightarrow k^{d}$ is etale.

Let

$$
V=\left\{(c, x) \in k^{r} \times X: c_{r}+\sum_{i=1}^{r-1} c_{i} s_{i}(x)=0\right\}
$$

The variety $V$ is smooth. Indeed, let $\left(g_{1}, \ldots, g_{r}\right)$ be the set of generators of the ideal $I(V) \subset k[X]\left[c_{1}, \ldots, c_{r}\right]$ and let $J(V)(c, x)=\left[\frac{\partial g_{i}}{\partial z_{j}}(c, x)\right]$, where $z=$ 
$\left(c_{1}, \ldots, c_{r}, x_{1}, \ldots, x_{d}\right)$ is a set of global local coordinates on $k^{r} \times X$. Let us note that a polynomial $h=\sum_{i=1}^{r} c_{i} s_{i}(x)$ does belong to the ideal $I(V)$. Now we see that rank $J(V) \geq 1$, because partial derivatives of $h$ with respect to $c_{i}$ form a matrix $\left[s_{i}(x)\right]_{1 \leq i \leq r}$, which has a rank 1 . Hence $\operatorname{dim} T_{(c, x)} V \leq d+r-1$. On the other hand $\operatorname{dim} V=d+r-1$ and consequently we have the equality $\operatorname{dim} T_{(c, x)} V=d+r-n=\operatorname{dim} V$.

Consider the projection:

$$
q: V \in(c, x) \rightarrow c \in k^{r}
$$

We show that for generic $c \in k^{r}$ the mapping $q$ is smooth on the set $q^{-1}\left(U_{c}\right)$, where $U_{c}$ is suitable neighborhood of $c$.

Indeed, let us compute the tangent space at $(c, x)$. It is given by the equation

$$
d c_{r}+\sum_{i=1}^{r-1} s_{i}(x) d c_{i}+\sum_{j}\left(\sum_{i}^{r-1} c_{i} \frac{\partial s_{i}(x)}{\partial x_{j}}\right) d x_{j} .
$$

Let us note that $\operatorname{rank}\left[\frac{\partial s_{i}(x)}{\partial x_{j}}\right]_{1 \leq i \leq r-1,1 \leq j \leq d}=d$. Indeed the mapping $\Phi: X \ni x \rightarrow$ $\left(s_{1}(x), \ldots, s_{r-1}(x)\right) \in k^{r-1}$ is unramified.

Put $L_{c}:=\left\{x \in X: \sum_{i=1}^{r-1} c_{i} \frac{\partial s_{i}(x)}{\partial x_{j}}=0, j=1, \ldots, d\right\}$. Let us note that the mapping $q$ is not a submersion in a neighborhood of $q^{-1}(c)$ a exactly if $L_{c} \neq \emptyset$. We show that the set $S:=\left\{c \in k^{r}: L_{c} \neq \emptyset\right\}$ is a constructible subset of $k^{r}$ of dimension less than $r$.

Indeed, let $W=\left\{\left(c^{\prime}, x\right) \in k^{r-1} \times X: \sum_{i=1}^{r-1} c_{i} \frac{\partial s_{i}(x)}{\partial x_{j}}=0, j=1, \ldots, d\right\}$. Let $\pi: W \ni(c, x) \rightarrow x \in X$. Let us note that the fiber of $\pi$ is a linear subspace of $k^{r-1}$ of dimension $r-1-d$ ( as a kernel of a suitable linear mapping). Hence $\operatorname{dim} W \leq r-1$.

Let $W^{\prime}=\left\{(c, x) \in V: \sum_{i=1}^{r-1} c_{i} \frac{\partial s_{i}(x)}{\partial x_{j}}=0, j=1, \ldots, d\right\}$. We have a surjective mapping

$$
s: W \ni\left(c^{\prime}, x\right) \rightarrow\left(\left(c^{\prime},-\sum_{i=1}^{r-1} c_{i}^{\prime} s_{i}(x)\right), x\right) \in W^{\prime} .
$$

Since $S=\rho\left(W^{\prime}\right)$, where $\rho: W^{\prime} \ni(c, x) \rightarrow c \in k^{r}$ and $\operatorname{dim} W=\operatorname{dim} s(W)=$ $\operatorname{dim} W^{\prime}$ we have $\operatorname{dim} c l(S) \leq r-1$.

This means that the projection $q: V \rightarrow k^{r}$ is a submersion outside a proper algebraic subset $\bar{S} \subset k^{r}$. In particular the zero set of a generic section $s=\sum_{i=1}^{r} c_{i} s_{i}$ is smooth.

Using a similar method we can generalize this result to higher dimension. As a Corollary we obtain Atiyah-Serre Spliting Theorem.

Theorem 3.2 Let $X$ be an algebraic variety of dimension $d$ and let $\mathcal{F}$ be a locallyfree sheaf on $X$ of rank $n$. Assume that $\mathcal{F}$ is generated by global sections $s_{1}, \ldots, s_{r} \in$ $\Gamma(X, \mathcal{F})$. Then there is a Zariski open non-empty subset $U \subset k^{r}$ such that for every 
$c=\left(c_{1}, \ldots, c_{r}\right) \in U$ the zero set of the section $s=\sum_{i=1}^{r} c_{i} s_{i}$ is either empty or it has dimension $d-n$. Moreover, if char $k=0$ and $X$ is smooth, then a generic section $s=\sum_{i=1}^{r} c_{i} s_{i}$ is transversal to $X$.

Proof Since $X$ is quasi-compact we can assume that $X$ is affine and the sheaf $\mathcal{F}$ is trivial, i.e., we can identify $\mathcal{F}$ with $\mathcal{O}_{X}^{n}$. Moreover we can assume that on $X$ we have a global local coordinates $\left(x_{1}, \ldots, x_{d}\right)$. Hence $s_{i}=\left(s_{i 1}, \ldots, s_{i, n}\right): X \rightarrow k^{n}$ are regular mappings. Let

$$
V=\left\{(c, x) \in k^{r} \times X: \sum_{i=1}^{r} c_{i} s_{i}(x)=0\right\} .
$$

Let us note that $\operatorname{dim} V=r+d-n$, where $d=\operatorname{dim} X$. Indeed, we have a surjection $\pi: V \ni(c, x) \rightarrow x \in X$. Any fiber of $\pi$ is a linear subspace of $k^{r}$ of dimension $r-n$ (it is a kernel of surjective linear mapping $F_{x}: k^{r} \ni c \rightarrow \sum_{i=1}^{r} c_{i} s_{i}(x) \in \mathbf{E}_{x}^{n}$, where $\mathbf{E}^{n}=X \times k^{n}$ is a trivial vector bundle of rank $n$ ). Consequently $\operatorname{dim} V=$ $\operatorname{dim} X+r-n=d+r-n$. Now consider the second projection:

$$
q: V \in(c, x) \rightarrow c \in k^{r}
$$

If it is dominated then the generic fiber has dimension $\operatorname{dim} V-\operatorname{dim} k^{r}=d+r-n-r=$ $d-n$, otherwise the generic fiber is empty.

Moreover, if $X$ is smooth over $k$, then $V$ is smooth. Indeed, let $\left(g_{1}, \ldots, g_{r}\right)$ be the set of generators of the ideal $I(V) \subset k[X]\left[c_{1}, \ldots, c_{r}\right]$ and let $J(V)(c, x)=\left[\frac{\partial g_{i}}{\partial z_{j}}(c, x)\right]$, where $z=\left(c_{1}, \ldots, c_{r}, x_{1}, \ldots, x_{d}\right)$ is a set of global local coordinates on $k^{r} \times X$. Let us note that polynomials $h_{j}=\sum_{i=1}^{r} c_{i} s_{i j}(x)$ does belong to the ideal $I(V)$. Now we see that rank $J(V) \geq n$, because partial derivatives of $h_{j}$ with respect to $c_{i}$ form a matrix $\left[s_{i j}(x)\right]_{1 \leq i \leq r, 1 \leq j \leq n}$, which has a rank $n$. Hence $\operatorname{dim} T_{(c, x)} V \leq d+r-n$. On the other hand $\operatorname{dim} V=d+r-n$ and consequently we have the equality $\operatorname{dim} T_{(c, x)} V=$ $d+r-n=\operatorname{dim} V$. If additionally char $k=0$, then the generic fiber is also smooth (generic smoothness-see [2], Corollary 10.7, p. 272).

Corollary 3.3 (Atiyah-Serre Splitting Theorem) Let $X$ be an algebraic variety of dimension $d$ and let $\mathcal{F}$ be a locally free sheaf on $X$ of rank $n$. Assume that $\mathcal{F}$ is generated by global sections $s_{1}, \ldots, s_{r} \in \Gamma(X, \mathcal{F})$. If $n>d$ then $\mathcal{F}$ contains a trivial subsheaf $\mathcal{A} \subset \mathcal{F}$ of rank $n-d$.

Proof Indeed, by induction we can find $n-d$ linearly independent sections $t_{j}=$ $\sum_{i=1}^{r} c_{j, i} s_{i}$ (note that the quotient sheaf of $\mathcal{F}$ is generated by the same sections (or rather their classes) as $\mathcal{F}$ ).

In particular if we assume that $X$ is affine we have the Serre result in a classical form:

Corollary 3.4 (Serre splitting theorem) Let $X$ be an affine algebraic variety of dimension $d$ and let $\mathcal{F}$ be a locally free sheaf on $X$ of rank $n$. If $n>d$ then $\mathcal{F}$ contains $a$ locally free subsheaf $\mathcal{F}^{\prime}$ of rank $d$ such that

$$
\mathcal{F}=\mathcal{F}^{\prime} \oplus \mathcal{O}_{X}^{n-d}
$$


Proof From the previous statement we know that $\mathcal{O}_{X}^{n-d} \subset \mathcal{F}$. Take $\mathcal{F}^{\prime}=\mathcal{F} / \mathcal{O}_{X}^{n-d}$. It is easy to see that $\mathcal{F}^{\prime}$ is also locally free sheaf of rank $d$. Moreover we have a short exact sequence

$$
0 \rightarrow \mathcal{O}_{X}^{n-d} \rightarrow \mathcal{F} \rightarrow \mathcal{F}^{\prime} \rightarrow 0
$$

This gives the following exact sequence

$$
0 \rightarrow \Gamma\left(X, \mathcal{O}_{X}^{n-d}\right) \rightarrow \Gamma(X, \mathcal{F}) \rightarrow \Gamma\left(X, \mathcal{F}^{\prime}\right) \rightarrow H^{1}\left(X, \mathcal{O}_{X}^{n-d}\right)=0
$$

Since $k[X]$ modules $\Gamma\left(X, \mathcal{O}_{X}^{n-d}\right), \Gamma(X, \mathcal{F}), \Gamma\left(X, \mathcal{F}^{\prime}\right)$ are projective (it is true locally hence also globally!) we have

$$
\Gamma(X, \mathcal{F})=\Gamma\left(X, \mathcal{F}^{\prime}\right) \oplus \Gamma\left(X, \mathcal{O}_{X}^{n-d}\right)
$$

Finally

$$
\Gamma(X, \mathcal{F})^{\sim}=\Gamma\left(X, \mathcal{F}^{\prime}\right)^{\sim} \oplus \Gamma\left(X, \mathcal{O}_{X}^{n-d}\right)^{\sim}
$$

and we have

$$
\mathcal{F}=\mathcal{F}^{\prime} \oplus \mathcal{O}_{X}^{n-d}
$$

(see [2], Prop. 5.1, p. 110).

Open Access This article is distributed under the terms of the Creative Commons Attribution License which permits any use, distribution, and reproduction in any medium, provided the original author(s) and the source are credited.

\section{References}

1. Atiyah, M.F.: Vector bundles over an elliptic curve. Proc. Lond. Math. Soc. 7, 414-452 (1957)

2. Hartshorne, R.: Algebraic Geometry. Springer, New York (1987)

3. Hartshorne, R.: Ample Subvarieties of Algebraic Varieties. Springer, Berlin (1986)

4. Kleiman, S.L.: The transversality of a general translate. Compositio Math. 28, 287-297 (1974)

5. Kleiman, S.L.: Geometry of grasmannians and applications to splitting bundles and smoothing cycles. Publ. Math. IHES 36, 281-297 (1969)

6. Serre, J.P.: Modules projectifs et espaces fibrés a fibre vectorielle. Sem. Dubreil-Pisot, vol. 2, Paris (1957/1958) 